\title{
La escuela y los alumnos con discapacidad motriz
}

\author{
Emili Soro
}

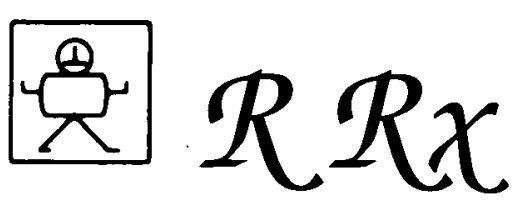

La educación normalizada de alumnos y alumnas con discapacidad motora es un tema actual. Desde una perspectiva psicoeducativa y habilitadora el artículo expone diversos problemas y sugerencias sobre criterios para la escolarización, objetivos prioritarios y estrategias metodológicas para la intervención y evaluación educativa.

\section{VALORACION DE LAS NECESIDADES EDUCATIVAS}

Las personas con discapacidad motora presentan características clínicas y funcionales muy distintas. Su discapacidad puede ser de origen congénito como la parálisis cerebral o la espina bífida, por causas adquiridas como los traumarismos craneales, por enfermedades degenerativas como algunas distrofias musculares, y su prevalencia puede ser permanente o temporal. La mayoría de estas afectaciones dificultan o imposibilitan la movilidad funcional de una o varias partes del cuerpo. Esta disfunción orgánica da lugar a clasificaciones como hemiplejía, paraplejía, diplejía, tetraplejía, etc., y a diferentes grados de disfunción muscular que pueden producir espasticidad, atetosis, ataxia, etc. En algunos casos la discapacidad conlleva otros trastornos asociados como son dificultades visuales, auditivas, neuropsicológicas, cognitivas, etc. (ver, entre otros, Basil, 1990; Bustos, 1984; Harmony y Alcazar, 1987; Ponces, 1991; Rapin, 1987).

Las categorías clínicas mencionadas no son explicativas de las limitaciones o posibilidades que presentan las personas con discapacidad. Por supuesto que para los profesionales de la educación interesa conocer el diagnóstico clínico de los alumnos/as, pero resulta mucho más constructivo valorar las necesidades educativas a partir del nivel funcional de la persona con discapacidad motora, de las posibilidades que ofrece la habilitación de la persona y del entorno con el uso de ayudas técnicas, y valorar los recursos profesionales con los que se cuenta. Es decir, valorar las necesidades educativas en términos de qué puede hacer el alumno/a, cómo puede realizar una tarea, cuanto tiempo puede centrarse en una actividad, con qué instrumentos puede mejorar su ejecución, en qué condiciones es más favorable que aprenda, con qué estrategias es más adecuado enseñar (Brown, 1989). Las necesidades educativas de estas personas pueden ser muy variadas y particulares. Estarán escolari- 
zados alumnos/as con disfunción motora muy leve hasta alumnos/as con alteraciones motoras muy graves que les impiden realizar cualquier movimiento voluntario; alumnos/as con una inteligencia normal o superior hasta alumnos/as con un retraso mental grave (ver, entre otros, Aguado y Alcedo, 1990; García, 1986).

\section{CRITERIOS PARA SU ESCOLARIZACION}

Todos los niños con discapacidad motora son candidatos a recibir una respuesta global de servicios, entre los cuales se debe introducir de manera precoz la atención educativa y escolar. Es cierto que en algunos casos y durante períodos determinados de su vida, el niño o joven con discapacidad motora puede necesitar mayor atención médica u otros servicios, pero esto nunca debería ser motivo para evitar la escolarización o reducir su asistencia escolar. En todos los casos los profesionales tendrán que proponer alternativas y medios diferentes a los tradicionales para conseguir objetivos educativos parecidos a los habituales, y cuando sea necesario se deberán proponer objetivos diferentes, adecuados a la normalidad del alumno.

Con la finalidad de establecer un relación orientativa entre la respuesta educativa que podrían recibir los alumnos y sus características funcionales de discapacidad se propone la agrupación siguiente.

Alumnos con discapacidad motora con inteligencia normal. Son alumnos que siguen el currículum ordinario pero que necesitan de medios especiales materiales, de instrumentos para el acceso alternativo a este currículum y/o requieren la omisión de determinados contenidos curriculares.

Alumnos con discapacidad motora y retraso mental moderado o discapacidad sensorial asociada (plurideficiència). Son alumnos que siguen una programación que incluye los núcleos fundamentales del currículum ordinario pero que presentan un ritmo de aprendizaje lento, requieren actuaciones educativas diferentes de las habituales, y necesitan disponer de medios específicos materiales, e instrumentos para el acceso alternativo a este currículum.

Alumnos con discapacidad motora y retraso mental grave y/o discapacidad sensorial asociada (plurideficiencia). Son alumnos que siguen un currículum relativamente alejado del ordinario. Los contenidos estan referidos, principalmente, a los hábitos de autonomía personal, a la comunicación, especialmente con medios técnicos y estrategias alternativos, y a las habilidades preocupacionales. En algunos casos la respuesta educativa va dirigida a resolver las funciones personales de la vida diaria y a garantizar un entorno de cualidad, tanto del alumno como de las personas que lo rodean.

En general, los alumnos pueden ubicarse en entornos escolares ordinarios, especiales o combinación de ambos. En la decisión del tipo de escolarización para los alumnos con discapacidad motora intervienen multitud de variables entre las cuales se han destacado las características de la persona, pero también debe considerarse las características del centro escolar, la disponibilidad de ayudas técnicas, la preparación de los profesionales, la opinión de la familia, etc. Por lo tanto, en cada caso y en cada momento se ha de analizar y valorar cuál es el entorno educativo más favorable.

\section{CONTENIDOS Y OBJETIVOS PRIORITARIOS}

Alumnos/as con imposibilidad o dificultad de realizar movimientos funcionales están expuestos a una mayor dependencia de los demás y ven comprometida la cantidad y calidad de experiencias que pueden producirse en el entorno escolar. Las 
necesidades educativas de estos alumnos implican, con frecuencia, una adecuación curricular de contenidos, objetivos y estrategias que garanticen la mejor accesibilidad posible a la educación normalizada (Brennan, 1988; Giné, 1987). Junto con la rehabilitación física dirigida específicamente a la persona, es importante habilitar el entorno para que se pueda conseguir, aunque de manera adaptada, el acceso a los contenidos y objetivos escolares. La adaptación del entorno y de los medios puede ser necesario durante todo el proceso educativo, pero cabe señalar algunas condiciones prioritarias como son el desplazamiento, la manipulación y el control de la postura.

El desplazamiento. Con la eliminación de barreras arquitectónicas y adaptación de las superficies de la escuela se garantiza que la mayoría de alumnos con dificultades motoras puedan acceder a todos los espacios de un edificio escolar. Otra forma de reducir barreras físicas es facilitar, cuando es necesario, el uso de bastones, caminadores, sistemas de desplazamiento asistido como una silla de ruedas autoimpulsada o una silla electrónica (Ver, entre otros, Hale, 1985; Otto Bock, 1989). La orientación y uso de estos instrumentos se hará de común acuerdo entre los diferentes profesio- nales implicados, pero en ningún caso se debería obviar que el objetivo general es que el alumno se desplace lo antes posible y de la manera más autónoma. Por ejemplo, Vicente es un niño de once años que en superficies llanas y para distancias cortas se desplaza gateando o de pie apoyándose en muebles; en el exterior utiliza un caminador frontal o una silla de ruedas mecánica autoimpulsada; para las excursiones usa una silla de ruedas electrónica.

FIGURA 1

Ayuda técnica diseñada para facilitar el desplazamiento asistido. Está impulsda por un motor eléctrico y dispone de adaptaciones para su manipulación y control

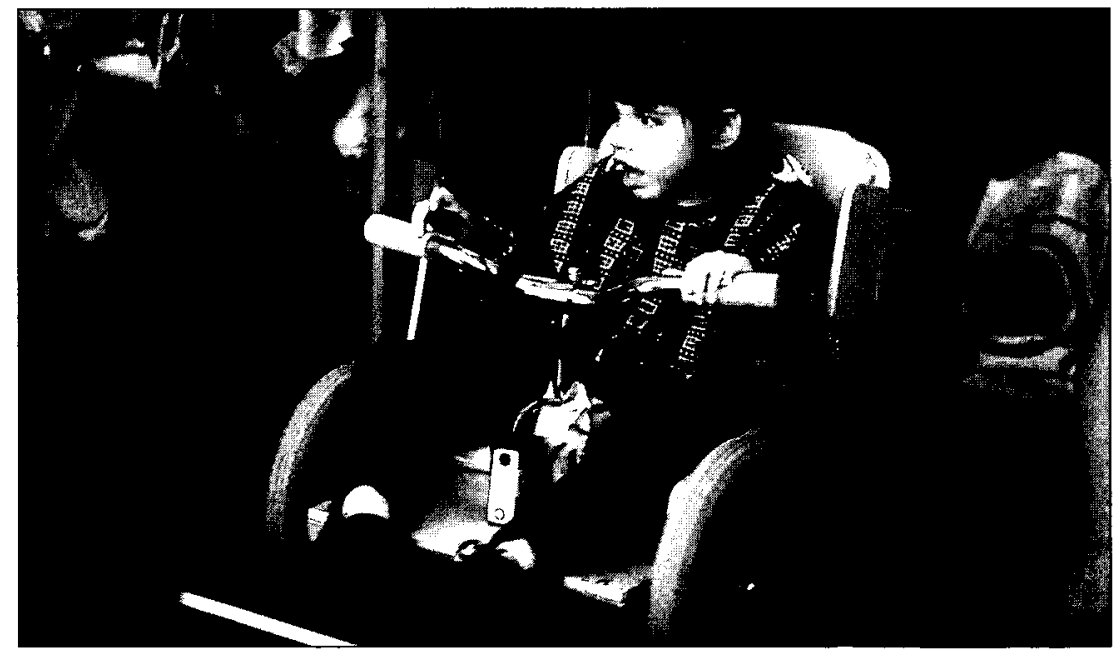

La manipulación. Muchos profesionales de la educación expresan su mayor preocupación cuando el alumno tiene dificultades en manipular objetos, asir un lápiz o pasar las páginas de un libro. Desde una perspectiva tradicional se diseñan y aplican procedimientos para que el alumno pueda practicar repetidamente hasta conseguir que realice las tareas motrices con la misma precisión y tiempo que los demás. En muchos casos esta práctica es adecuada y necesaria. Pero no siempre es correcto supeditar la forma tradicional de realizar una actividad gráfica a los contenidos que se pueden enseñar y aprender, aunque éstos se realicen de una manera alternativa. Algunos alumnos/as, desde los primeros momentos en que pueden acceder a actividades gráficas de dibujo o escritura pueden combinar el uso de lápices adapta- 
dos con ordenadores dotados de programas de juegos, dibujo y texto, o otros instrumentos (Gomez, Alamillo y Junoy, 1988). Por ejemplo, Juan es un niño de ocho años, con una distonía que le impide realizar caligrafía legible. Se prevee que podrá mejorar su habilidad de prensión y precisión con la mano. Pero actualmente ya usa un adaptador que aumenta el volumen del lápiz, un tejido adherido sobre la mesa que frena sus movimientos involuntarios, y un ordenador con teclado normal.

Figura 2

El alumno puede realizar actividades gráficas con la ayuda de un ordenador que le permite controlar un programa mediante la activación de dos conmutadores

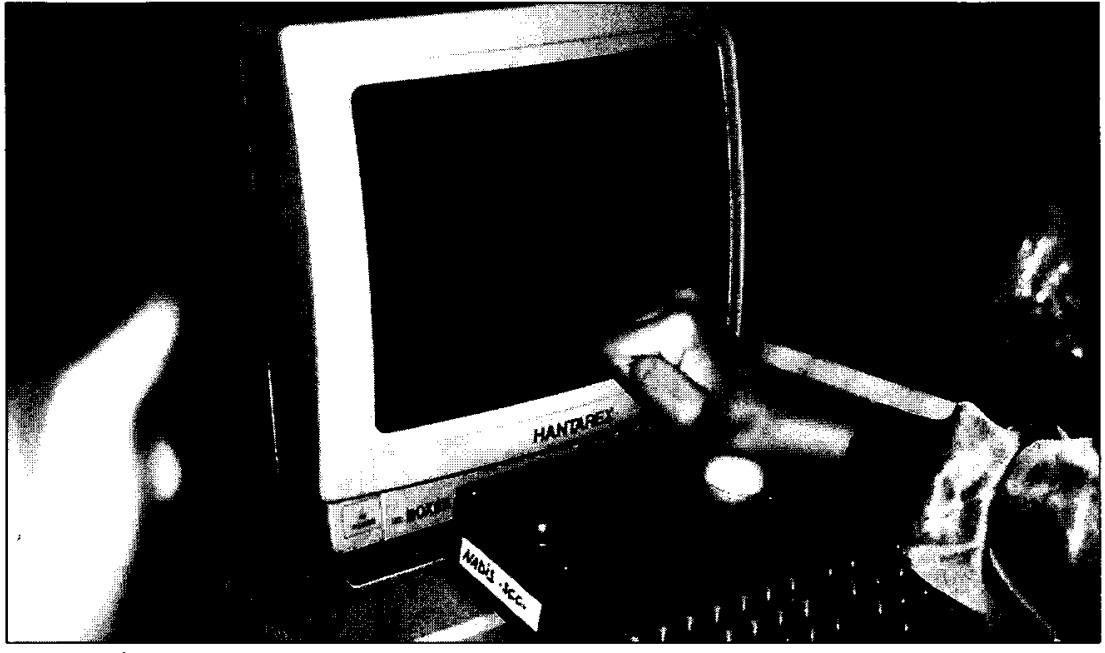

El control de la postura. Es importante que la postura física del alumno sea lo más correcta posible, tanto en condiciones de sedestación como en bipedestación o desplazamiento. Una buena postura corporal es importante para prevenir malformaciones óseas, evitar cansancio muscular, mejorar la percepción y realización de las tareas (McEwen y Karlan, 1989; Otto Bock, 1989). Para garantizar el beneficio del control de la postura y evitar que éste no interfiera en la calidad de las tareas escolares el profesional de la educación necesitará la colaboración de fisioterapeutas o terapeutas ocupacionales expertos. En ocasiones el control postural se puede mejorar a través de técnicas de autocontrol que aprende la persona, en otras se ha de modificar el mobiliario o los intrumentos que han de usar. Por ejemplo, Miguel es un joven de quince años con parálisis cerebral que cuando ha de utilizar papel y lápiz durante un periodo largo se requiere una silla con elevación del lado izquierdo para evitar la inclinación involuntaria del cuerpo, un soporte sobre la mesa para fijar su mano izquierda, y un reposapies con el fin de evitar movimientos asociados.

Las condiciones hasta aquí mencionadas constituyen una respuesta a las necesidades físicas de algunos alumnos con discapacidad motora, pero también constituyen un medio habilitador para optimizar sus posibilidades de participación activa en el contexto escolar, y por lo tanto acceder con mayor facilidad a los contenidos y objetivos prioritarios durante la escolarización, entre los cuales se destacan los que, en general, requieren una mayor adecuación individualizada.

Comunicación y lenguaje. El alumno/a con un trastorno motor puede presentar diversas dificultades en la comunicación y el lenguaje (ver, entre otros, Puyuelo, 1982a, 1982b, 1986; Schiefelbus, 1986) pero lo que mayoritariamente llama la atención es la dificultad o imposibilidad de usar el habla. En muchos casos parece 
más indicado poner el énfasis en los objetivos dirigidos al uso y contenido del lenguaje, para preocuparse posteriormente de la forma lingüística (Soro y Edo, 1988). Cuando los problemas motores interfieran en la inteligibilidad del habla o en el acceso a la lectura y la escritura se establecerán procedimientos de rehabilitación del habla con o sin el soporte de sistemas signados o quinestésicos de apoyo. Por ejemplo, Laura es una niña de siete años que habla con más seguridad e inteligibilidad cuando se ayuda de movimientos con una mano para marcar las sílabas. Cuando existen trastornos específicos del lenguaje, y no solamente del acto motor del habla, que afectan tanto la expresión como la comprensión del lenguaje se requerirán programas dirigidos a crear y potenciar las bases de la interacción (ver, entre otros, kent, 1983, Rondal, 1988). Cuando los alumnos no pueden usar el habla será imprescindible organizar el uso de sistemas aumentativos y alternativos de comunicación. En este caso los contenidos y objetivos, tanto los referidos a los alumnos como los referidos a los interlocutores, tendrán que adaptarse a las características de los medios de comunicación que se dispongan: tableros de comunicación, máquinas para hablar, ordenadores, etc. (ver, entre otros, Basil y Ruiz, 1984; Puig de la Bellacasa y Sánchez de Muniaín, 1988; Sanchez de Munianín, Castro, Fernández y Castellanos, 1989).

En cuanto el aprendizaje y uso de la comunicación escrita, siempre que sea posible, es recomendable facilitar precozmente al alumno/a el acceso a la lectura y la escritura. Ayudas técnicas para pasar las páginas de los cuentos, útiles para ejecutar grafías con un mínimo de esfuerzo, etc. pueden ser la mejor forma de desarrollar los objetivos de acceso a la lectura y la escritura (Hilmerson, 1984, 1986).

FIGURA 3

Con la adaptación de sellos de goma una alumna puede estampar dibujos, letras, palabras, etc.

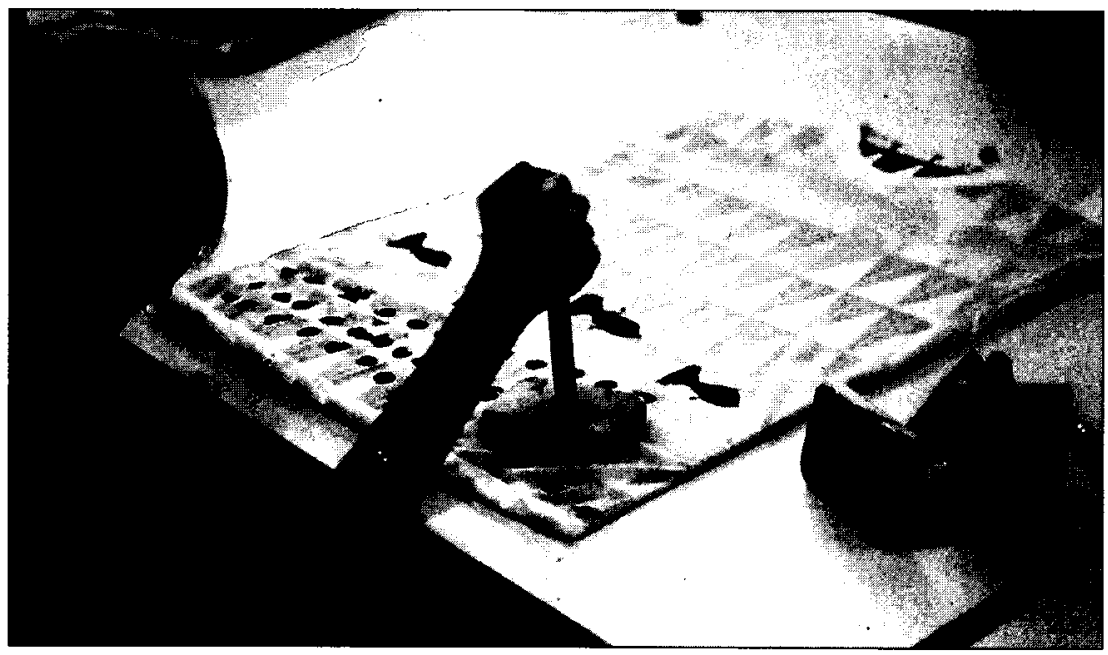

Las habilidades de autonomía de trabajo. El alumno con discapacidad motora puede ver muy comprometida su autonomía de trabajo en el contexto de una aula, y necesitar la constante ayuda de una maestra de soporte. Con frecuencia, si los profesionales diseñan una área adaptada de trabajo, el alumno será más autónomo en la realización de las tareas, más independiente de los adultos y otros compañeros, y los profesionales podrán dedicar su tiempo a la programación de contenidos y procedimientos de enseñanza. Por ejemplo, Isabel es una niña de ocho años, sigue el curso escolar normal y puede realizar la mayoría de las actividades escolares sin ayuda, pero con la siguiente organización: la alumna está situada cerca de la pizarra y junto a una mesa de servicio sobre la que estan la mayor parte de los materiales que se usan (tije- 
ras, colores, papel, fichas, libros, etc.). La mesa de la alumna se adaptó con protecciones laterales, casilleros verticales en la parte frontal, separadores de carpetas a su alcance para la distribución y entrega de tareas, etc.

FIGURA 4

El alumno pasa las páginas colocadas en el "girapáginas» eléctrico, al activar un conmutador situado sobre su mesa

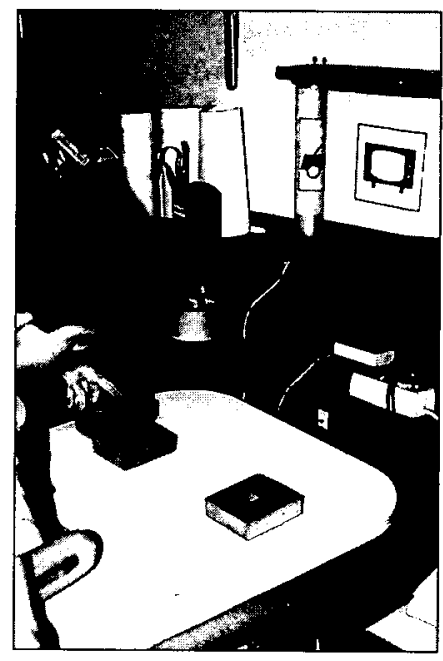

Las babilidades de autonomía personal. Los objetivos centrados en alimentación, vestirse y desvestirse y control de esíinteres deberían ser prioritarios y tratados con precocidad en todos los casos. Tan desacertado puede ser considerar estos objetivos como los únicos relevantes, como no dedicarles el tiempo necesario por la dificultad que encierran. Existen diversos materiales adaptados para facilitar que un niño coma solo, y cuando ésto no es posible es importante convertir esta actividad, ya de por sí difícil para el niño, en un tiempo de interacción relajada. Para facilitar la autonomía en el vestirse se puede recurrir a prendas adaptadas y formas atípicas de vestirse. $Y$ en cuanto al control de esfínteres, exceptuando los casos en que se ha comprobado una lesión orgánica, los objetivos pueden ser los mismos que se marcarían con otros niños, pero prescindiendo de los objetivos que implican manipulación o desplazamiento, cuando ésto es imposible.

En la consecución de estos contenidos y objetivos se veran implicados profesionales de diferentes áreas: educación, fisioterapia, neuromedicina, psicología, logopedia, informática, electrónica, asistencia social, etc., y por supuesto la propia familia. La interrelación de todas las disciplinas e intereses que pretenden ayudar al alumno con discapacidad no es nada fácil, pero es importante encontrar modelos de coordinación que hagan el trabajo agradable, ágil y que garanticen que el objetivo último es que el niño, al igual que todos los demás, logre desarrollar al máximo sus capacidades para lograr una vida de relación y aprovechamiento de su tiempo de trabajo y de ocio lo más rica, adaptada y feliz posible, sin tener que convertirse en en verdadero «hiperconsumidor» de programas de rehabilitación.

\section{ESTRATEGIAS METODOLOGICAS Y ORGANIZATIVAS}

Responder a la diversidad de necesidades educativas de los alumnos con discapacidad motora, estén situados en entornos ordinarios o especiales, no es una tarea 
fácil. Profesionales con una actitud favorable a la normalización escolar y social de todos los alumnos, modelos de programación, recursos personales y materiales son condiciones necesarias pero no suficientes para garantizar un entorno educativo de calidad. Es importante crear un entorno que optimice las condicones de enseñanza y aprendizaje de alumnos con discapacidad motora, es decir, un marco educativo adaptado a las necesidades normales de los alumnos con discapacidad. Como han señalado Soro y Marco, 1990) para la organización de este entorno se pueden utilizar varias estrategias metodológicas. Estrategias referidas al espacio, a los materiales, a la organización y a los procesos de enseñanza.

Espacio. Cuando los alumnos utilizan ayudas técnicas como ordenadores, comunicadores, tableros plastificados, etc. es imprescindible cuidar los puntos de iluminación para que el alumno tenga luz suficiente o que la luz no provoque reflejos que le impidan ver con nitidez. Para facilitar el acceso a los materiales es imprescindible situar los armarios, estanterías, paneles de información, estímulos didácticos, pizarras, etc. a la altura de los ojos y de las manos de alumnos que utilizan sillas de ruedas. Con frecuencia es aconsejable crear espacios de trabajo individual, de manera que el alumno se pueda mover con facilidad, sin interferir con otros compañeros.

Materiales. Como ya se ha mencionado, el alumno con discapacidad motora puede encontrarse con limitaciones para explorar y manipular el entorno, para hablar, para escribir, para dibujar, ocupar su tiempo libre, etc. y esto puede significar, en muchos casos, que sin las ayudas técnicas y pedagógicas adecuadas esos alumnos no consigen actualizar sus potenciales intelectuales (Basil, 1990). La habilitación del alumno con discapacidad comporta el uso de múltiples ayudas técnicas que configuran una forma alternativa de acceso a los contenidos curriculares y a las actividades de la vida diaria. Para la correcta atención educativa es imprescindible disponer de ayudas técnicas generales e individuales adecuadas a les necesidades de cada alumno. Algunas ayudas técnicas pueden ser de uso común para varios usuarios, como es un cabezal-licornio, un puntero luminoso, un juego electrónico adaptado, etc., otras ayudas han de ser personalizadas, como un comunicador, un ordenador portátil. Estas ayudas deberían ser probadas antes de prescribirse a los alumnos, y ésto obliga a reflexionar sobre la importancia y urgencia de disponer de centros de recursos adecuados y de establecer una política racional que regule la adquisición, el uso y el reciclaje de las ayudas técnicas. El coste de materiales puede ser elevado, pero los recursos són una inversión imprescindible para ofrecer una respuesta educativa correcta a muchos alumnos/as con discapacidad motora.

Organización. La forma de organizar la globalidad de los servicios puede mejorar la intervención educativa. Centros de recursos debidamente equipados, con servicio de préstamo de materiales, atendidos por profesionales expertos que puedan asesorar a familias, usuarios y profesionales de atención directa, pueden ser la solución de algunos problemas. Adecuar modelos de interacción interprofesional que faciliten el diálogo y el intercambio de información sobre los objetivos y metodología que se va a utilizar en cada persona podría mejorar la atención en equipo entre familia, educadores y otros profesionales. Por otra parte, los medios de los centros de educación han de responder a las necesidades educativas especiales de los alumnos. La sectorización de servicios que permiten al alumno con discapacidad motora poder asistir a la escuela de su barrio o zona, cerca de casa y junto con sus compañeros de vida extraescolar es un acierto personal y social. La escuela ordinaria presenta ventajas de todo tipo para la mayoría de estos alumnos, para sus familias, y para sus compañeros de clase. Pero cuando esta escuela no puede garantizar al alumno con discapacidad la atención especial que necesita, 
quizás sea mejor solución ubicar al alumno con discapacidad, temporalmente o no, en un centro especializado, o compartir servicios entre centros especializados y ordinarios.

Proceso educativo. A nuestro entender, una de las estrategias metodológicas más importante radica en la habilidad o estilo de enseñar del profesional. Este, además de conocer los principios generales de procedimientos didácticos, pedagógicos y psicológicos, necesita conocer procedimientos y estrategias especiales que garantizen la adquisición de contenidos y objetivos curriculares. Las estrategias no son fórmulas mágicas que se pueden utilizar en todos los casos, en todas las áreas y en cualquier punto del programa educativo. Por lo tanto, la primera estrategia exige ser flexible y ágil en el uso de las mismas, otras pueden ser: (a) seleccionar objetivos funcionales y relacionados con la vida cotidiana del alumno/a, (b) secuenciar en pequeños pasos los objetivos que se han marcado, (c) practicar el suficiente número de veces para consolidar el aprendizaje, (d) establecer la duración de las tareas de acuerdo a la pauta de cansancio físico y el nivel de atención óptimo, (e) establecer el tipo de soportes verbales, manuales o materiales que sean necesarios y retirarlos progresivamente, cuando es posible, $(f)$ esperar el tiempo suficiente para que el alumno pueda contestar, $(\mathrm{g})$ ofrecer oportunidades para un mayor protagonismo en las interacciones en grupo, (h) diferenciar los distintos niveles de competencia que presenta el alumno en las diversas áreas o contenidos y establecer los grados de exigencia apropiados para cada nivel, etc. En Hinojosa y Galindo (1984) se desçriben algunas estrategias aplicadas en el área motora, en von Tetzchner (1993) se dedican varios capítulos a describir y ejemplificar estrategias metodológicas de intervención en entornos naturales, específicamente en el área de la comunicación y el lenguaje; Basil, Soro y von Tetzchner (1992) ilustran algunas de las estrategias que se pueden utilizar para la enseñanza y aprendizaje inicial de comunicación cuando el alumno no puede usar el habla. Como ya es sabido, en el proceso de enseñanza-aprendizaje intervienen multitud de variables, y a modo de ejemplo aquí se han mencionado algunas de ellas. Qué enseñar, cómo enseñar, con qué frecuencia, en qué situación, etc. son cuestiones metodológicas de máxima prioridad en la enseñanza.

\section{CRITERIOS Y PROCEDIMIENTOS PARA LA EVALUACION}

Desde una perspectiva educativa es apropiado que la evaluación se concrete y relacione con el modelo curricular de programación que se utiliza. La evaluación puede ser tan completa y compleja como se desee, pero el proceso de evaluación y los contenidos que se evaluan deben vincularse con los procesos de intervención educativa. Ruiz (1988) propone una terminología y modelo de Adecuación Curricular Individual (ACI), a partir del cual se puede organizar un esquema de intervención-evaluación individualizado. En Calvo, Gracia, Martín y Montero (1990, p. 58) se pueden encontrar algunos modelos de registros basados en un enfoque curricular. En la figura 5 se ilustra la adaptación de un registro para la programación y evaluación de contenidos (extraido de Soro, 1991). En él se concretan, de una forma clara en una misma página, los objetivos generales y específicos, el nivel de competencia actual del objetivo general, estrategias metodológicas que se utilizarán para enseñar los objetivos específicos, y criterios para la evaluación de los mismos.

El proceso de evaluación no ha de valorar solamente el alumno/a, sino que ha de ser, principalmente, la búsqueda de respuestas a las necesidades de cada persona y un medio para garantizar la eficacia de la intervención educativa, tomando en consi- 
A.C.I. CONTENIDO, OBJETIVO GENERAL Y OBJETIVOS ESPECIFICOS

ALUMNO/A

CURSO.

AREA:

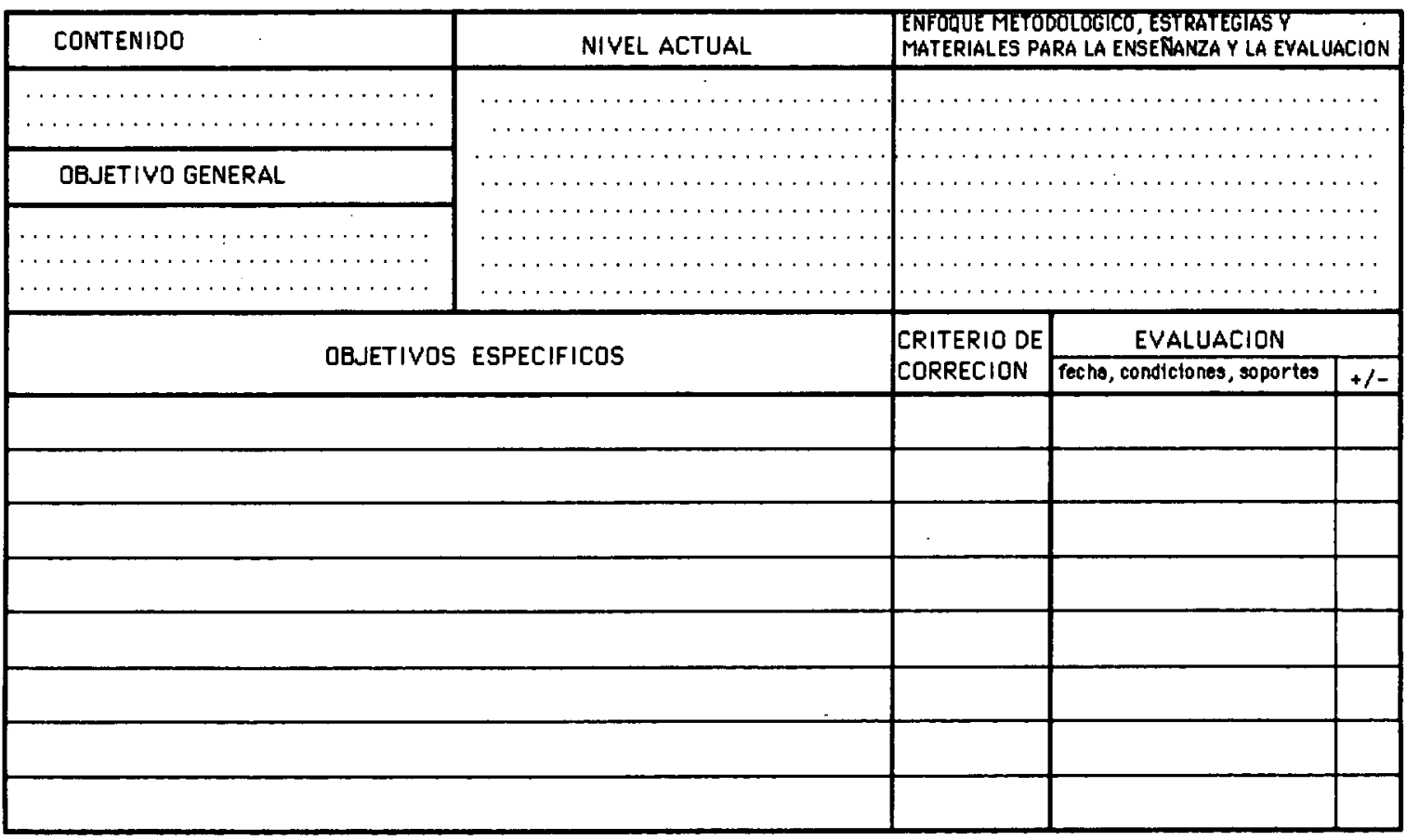

User una hoje para codo objetivo general. User registros especiales para los objetivos especificos que lo requieran. Notas al dorso. 
deración tanto el alumno como la situación general en la que éste se encuentra. En la evaluación se pueden contemplar varios aspectos:

Evaluación inicial de las habilidades del alumnola, y características del entorno. ¿Qué programas educativos ha seguido?, ¿Cómo valoran los padres los aprendizajes de su hijo/a?, ¿Cuál es su nivel escolar?, ¿Qué oportunidades tiene para aprender?.

Evaluación de los objetivos programados. ¿Los objetivos son adecuados al nivel del alumno/a?, ¿Los objetivos plantean una progresión de dificultad sensible a la capacidad del alumno/a?.

Evaluación de las estrategias, procedimientos, recursos, etc. que se ban programado. ¿Por qué no aprende el alumno/a?, ¿En qué falla el programa?, ¿Qué errores metodológicos se producen?

Evaluación de cómo y qué ba adquirido el alumnola. ¿Qué ritmo de adquisición presenta el alumno/a?, ¿Cómo se ajusta su adquisición respecto a la globalidad del programa?, ¿En qué condiciones aprende mejor?, ¿En qué medida generaliza sus adquisiciones?, ¿Qué nivel de conocimientos y habilidades ha adquirido?

Evaluación de la enseñanza. ¿Qué han aprendido otros alumnos en condiciones similares?, ¿Qué nivel ha conseguido el grupo?, ¿Qué he aprendido como maestro o profesional de la educación?.

Un esquema curricular puede ser el principal punto de referencia del proceso de evaluación educativa, pero no el único. En algunos casos puede ser necesario usar otros instrumentos de recogida de información que ayuden a elaborar, modificar y evaluar el programa educativo. Entre los instrumentos más utilizados destacan los que siguen.

Información escrita previa. Informes médicos, neurológicos o pedagógicos, programas educativos anteriores, etc.

Entrevistas orales con la familia y profesionales. El profesional puede utilizar modelos de entrevista con preguntas concretas, guiones con preguntas generales, etc., pero siempre que sea posible es interesante aprovechar el contexto de conversación informal. Esta información puede constituir la base de una evaluación formal o puede ser una complementación de los datos recogidos en los test o registros observacionales.

Registros de babilidades y comportamientos. A menudo son un conjunto de cuestiones que se han extraído de escalas evolutivas, protocolos de evaluación, inventarios ecológicos, diseños curriculares, etc., o pueden ser registros que el profesional ha construido a partir de la información recabada en las entrevistas o de la documentación escrita previa.

Tests psicopedagógicos. Como afirma von Tetzchner (1993), «una buena regla para el empleo de este tipo de pruebas es preguntarse siempre de antemano para qué se van a usar los resultados que se obtengan». En la evaluación educativa en personas con discapacidad motora los tests presentan algunas limitaciones: (a) en general no proporcionan información concreta sobre objetivos pedagógicos, (b) exigen la realización de tareas manuales y de comunicación oral o escrița, (c) los contenidos y resultados han estado estandarizados con poblaciones sin discapacidad motora. No obstante, la pasación de tests tradicionales es posible cuando se adapta el tiempo de pasación, el tipo de instrucciones, la manera cómo se presentan los estímulos, la forma de respuesta, etc. Aceptando estas alteraciones los tests puede proporcionar información útil derivada tanto del proceso de pasación como de las respuestas concretas, estos resultados pueden ser orientadores junto con los datos hallados con otros procedimientos.

Observación sistemática. Es importante observar a la persona en situaciones y actividades diferentes, pero es fundamental observar de forma sistemática. Las apreciaciones cualitativas espontáneas han de contrastarse con las observaciones cuantita- 
tivas, realizadas en varios días, en diferentes situaciones y con personas distintas. La tecnología del vídeo resulta útil porque facilita el archivo de las observaciones, verlas repetidamente, comparar con situaciones anteriores, compartir la opinión de diferentes personas, documentar el progreso de los niños, y son una buena base para ilustrar las entrevistas informativas y formativas con los padres.

Procedimiento de intervención-evaluación. Cuando la evaluación inicial no es posible o recomendable puede ser necesario un periodo de intervención durante el cual se practica una recogida progresiva de datos. A medida que el evaluador dispone de más información sobre el alumno, y éste adquiere más habilidades se podrá pensar en introducir métodos de evaluación más formales, si es necesario.

Además de los instrumentos mencionados, en la evaluación de alumnos/as con discapacidad motora también se necesitarán materiales adaptados o específicos como férulas, sillas para posicionamiento, comunicadores, conmutadores, indicadores con luz, pictografías, microordenadores, etc. (ver, entre otros, Hale, 1985; Gardeazabal, 1988). Las nuevas tecnologías son un excelente recurso para la evaluación de alumnos con discapacidad motora, especialmente los ordenadores que ofrecen versatilidad para la conexión de distintos periféricos, y que disponen de programas específicos para la evaluación. El uso de los instrumentos, adaptaciones y nuevas tecnologías deberá completarse con la investigación y aplicación de nuevos procedimientos y modelos de evaluación.

\section{Referencias}

Aguado, A., y AlCEDo, M. A. (1990). Las estadísticas españolas de minusválidos físicos: una revisión crítica. Análisis y Modificación de Conducta, 16(50), 507-548.

BASII, C. (1990). Los alumnos con parálisis cerebral: Desarrollo y educación. En A. Marchesi, J. Palacios, y C. Coll (Eds.), Desarrollo psicologico y educación III: Necesidades educativas especiales y aprendizaje escolar. Barcelona: Santillana.

BASIL, C.; SORO, E., y vON TETZCHNER, S. (1992). Estrategias iniciales para la enseñanza de comunicación assistida. (Video). (Se puede obtenr en: ADAB Agència Literaria, Còrsega 60 àtic, 08029 Barcelona). Barcelona: Universitat de Barcelona. ICE.

BASn, C., y RUIZ, R. (198). Sistemas de comunicación no vocal para niños con disminuciones físicas. (2a edición). Madrid: FUNDESCO.

BRENNAN, W. K. (1988). El currículo para niños con necesidades especiales. Madrid: M.E.C. / Siglo XXI de España Editores S.A.

BRown, L. (1989). Criterios de funcionalidad. Barcelona: Editorial Milán.

Bustos, C. (1984). Reeducación del habla y el lenguaje en el paralítico cerebral. (2 $2^{a}$ Edición). Madrid: CEPE.

Calvo, R. E.; Gracia, B.; Martin-Caro, L., y Montero, I. (1990). Evaluación. Adaptaciones para niños con deficiencias motoras (Tema Tres). En Serie Información. Las Necesidades Educativas Especiales del niño con Deficiencia Motora. Madrid: M.E.C. y C.N.R.E.E.

GARCIA, H. S. (1986). Deficientes físicos gravemente afectados. Estimación de la población existente. Minusval, 53, 32-35.

GARDEAZABAL, L. (1989). Aplicaciones del ordenador y de las nuevas ternologias en la ayuda a personas con discapacidad. San Sebastián: Servicio Editorial de la UPV-EHU.

GINE, C. (1987). El retraso en el desarrollo: una respuesta educativa. Infancia y Aprendizaje, 39-40, 8394.

GOMEZ, P. M. J.; AlammLo, S. S., y JuNOY, G. M. (1988). Posibilidades de aplicación de la informática a la educación especial. (Serie Orientaciones Pedagógicas 2.) Madrid: M.E.C. y C.N.R.E.E.

Harmony, T., y AlCaraz, V. M. (1987). Daño cerebral. Diagnóstico y tratamiento. México: Trillas.

Hilmersson, G. (1986). Escribir. (Gotenburgo:Centro de Rehabilitación Bräcke Ostergard). (Original de 1982). Madrid: ATAM-FUNDESCO.

HILMERSSON, G. (1984). Aprender a leer. (Gotenburgo: Centro de Rehabilitación Bräcke Ostegard). (Versió original de 1984. Traducción de M. Breien). Madrid: ATAM-FUNDESCO.

HINOJOSA, G., y GALINDO, E. (1984). La enseñanza de los niños impedidos. México: Trillas.

KENT, L. R. (1983). El niño que no se comunica: bases teóricas y prácticas para la intervención. Revista de Logopedia y Fonaaudiologia, 3, (2), 78-95.

MCEWEN, I. R., y KARLAN, G. R. (1989). Assessment of effects of position on communication boards access by individuals with cerebral palsy. Augmentative and alternative communication, 5, 235-242. 
OTто Bоск. (1989). Criterios de la sedestación. Tendencias actuales para los incapacitados. (Traducción de Loudes Tortosa i Alexander Ruth). Canadá: Otto Bock Orthopedic Industry of Canada Ltd.

PONCES, J. (1991). Parálisis cerebral infantil. Qué ens cal saber? Barcelona: Generalitat de Catalunya. Departament de Benestar Social.

Puig de la Bellacasa, R., y Sanchez de Muniain, P. (1988). Ayudas técnicas para la comunicación no-vocal. En C. Basil y R. Puig (Eds.), Comunicación aumentativa (pp. 49-82). Madrid: Colección Rehabilitación, INSERSO.

PUYutio, M. (1982a). Logopedia y parálisis cerebral infantil. Revista de logopedia y Fonaaudiología, 1, (4), 219-235.

PuYUELo, M. (1982b). El diagnóstico logopédico en la parálisis cerebral y el retraso psicomotor. Revista de Logopedia y Fonoaudiología, 2, (1), 10-16.

PUYUELO, M. (1986). Las sesiones de logopedia en la integración de la parálisis cerebral infantil. Revista de Logopedia y Fonoaudiología, 1, (6), 14-22.

RAPIN, I. (1987). Disfunción cerebral en la infancia. Barcelona: Martínez Roca.

RONDAL, J. A. (1988). Estrategias de enseñanza adoptadas por los padres y aprendizaje del lenguaje. Revista de Logopedia, Foniatría y Audiología, 8 (1), 11-22.

RuIZ, R. (1988). Técnicas de Individualización Didáctica. Adecuaciones Curriculares Individualizadas para alumnos con necesidades educativas especiales. Madrid: Editorial Cincel.

Sanchez de Muniain, P.; Castro, C.; Fernandez, M.; Castellanos, M. C., y Gonzalez, L. (1987). Las ayudas técnicas en los sistemas de comunicación aumentativa. Serie: Ayudas Técnicas. Documento I. Madrid: ATAM-FUNDESCO.

SCHIEFELBUSCH, R. L. (1986). Bases de la intervención en el lenguaje. Madrid: Alhambra.

SORO, E. (1992). Diseño curricular: proceso de evaluación e intervención. (Manuscrito en revisión editorial). La coruña: Fundación PAIDEIA.

SORO, E., y EDO, S. (1988). Aprendizaje de la emisión de enunciados de más de un elemento. Caso de un alumno no-vocal. Revista de Logopedia y Fonaaudiología, 8, (1), 30-40.

SORO, E., y MARCO, F. (1990). Sistemas aumentativos de comunicación como ayuda a la normalización escolar. En C.N.R.E.E. Las neresidades educativas especiales del niño con deficiencia motora. Seire Formación. Madrid: M.E.C. y C.N.R.E.E.

VON TETzCHNEr, S., y MARTINSEN, H. (1993). Introducción a la enseñanza de signos y al uso de ayudas térnicas para la comunicación. Madrid: Visor.

\section{Bibliografía básica. Sugerencia para los lectores}

Basil, C., y PUIG DE la Bellacasa, R. (Eds.). (1988). Comunicación aumentativa. Madrid: INSERSO.

BusTOs, C. (1984). Reeducación del habla y el lenguaje en el paralitico cerebral. (2a Edición). Madrid: CEPE.

C.N.R.E.E. (1990). Las necesidades educativas especiales del niño con deficiencia motora. Seire Formación. Madrid: Centro Nacional de Recursos para la Educación Especial.

CASCON, P., y MARTIN, C. (Eds.). (1989). La alternativa del juego. Fichas térnicas. (8a. Edició). (Se puede obtener en: Colectivo educar para la paz, Apdo. de Correos 219, Torrelavega, Cantabria, España) Cantabria: Colectivo educar para la paz.

FINNE, R. N. (1987). Atención en el bogar del niño con parálisis cerebral. Madrid: La Prensa Médica Mejicana.

HALE, (1985). Manual para el minusválido. Barcelona: Blume.

MUSSEL WHITE, C. R. (1990). Juegos adaptados para niños con necesidades especiales. Madrid: INSERSO. Colección Rehabilitación, 34.

Soro, E.; Rosell, C.; Alsina, G.; Garcia, H.; SAnChez de Munian, P.; Comellas, A.; Vilaseca, R. M., y Edo, S. (1988). Manual de toma de decisiones y de evaluación para el aprendizaje y uso de los sistemas aumentativos de comunicación. Serie: evaluación. documento I (Se puede obtener en: Nadís, Canàries 1, 08017 Barcelona). Madrid: ATAM-FUNDESCO.

Toledo, M. (1984). La escuela ordinaria ante el niño con nexesidades especiales. Madrid: Santillana-Aula XXI.

VON TETZCHNER, S., y MARTINSEN, H. (1993). Introducción a la enseñanza de signos y al uso de ayudas técnicas para la comunicación. Madrid: Visor. 


\section{La escuela y los alumnos con discapacidad motriz Emili Soro CL\&E, 1994, 22, pp. 23-35}

Resumen: En este artículo se resalta la importancia de evaluar las necesidades educativas de los niños con deficiencias motrices, y se resalta el papel del entorno educativo (personas y recursos técnicos) a la hora de abordar diseños específicos de actuación. Se propone un modelo curricular basado en esos requisitos y se describen los procedimientos y materiales utilizados.

Datos sobre el autor: Emili Soro-Camats es licenciado en Psicología y diplomado en Logopedia. Actualmente trabaja en la Fundació l'Espiga y en la Unidad de Técnicas Aumentativas de Comunicación; ambas entidades orientadas a la atención de personas con discapacidad.

Dirección: Eugeni D'Ors s/n, 08720 Vilafranca del Penedès. Barcelona.

(C) PERMISOS PARA CITAR O REPRODUCIR EN OTRAS FUENTES: Se pueden citar libremente hasta 500 palabras. Para reproducir una porción de texto mayor, figuras o ilustraciones, se deberá pedir permiso por escrito a la revista, especificando el uso al que se destina el texto. En todos los casos, se deberá citar el copyright de $C L \& E$. En el caso de artículos o textos que hayan sido a su vez reproducidos en $C L \& E$ los interesados deberán dirigirse tanto a los detentadores del copyright original como a $C L \& E$, en el caso de que se quiera hacer uso de la traducción. FOTOCOPIAS: Para todo lo relacionado con el uso mediante fotocopia del material de esta revista, deberán dirigirse a: CEDRO, C/ José Marañón, 10, $3 .^{\circ}$ Izda. Tel. 5941575 . Fax 4453567 